\title{
Erratum to: Arsenic Concentrations and Associated Health Risks in Laccaria Mushrooms from Yunnan (SW China)
}

\author{
Ji Zhang ${ }^{1} \cdot$ Tao Li $^{2}$ - Ya-Li Yang ${ }^{2} \cdot$ Hong-Gao Liu ${ }^{3} \cdot$ Yuan-Zhong Wang ${ }^{1}$
}

Published online: 17 April 2015

(C) Springer Science+Business Media New York 2015

Erratum to: Biol Trace Elem Res (2014)

DOI 10.1007/s12011-014-0213-3

The article "Arsenic Concentrations and Associated Health Risks in Laccaria Mushrooms from Yunnan (SW China)", Biol Trace Elem Res, DOI 10.1007/s12011-014-0213-3 unfortunately contained mistakes. L. laccata ( $\equiv L$. amethystina) found in Table 2 (two occurrences) should read as $L$. laccata ( $\equiv L$. amethystea). The correct table is shown below.

The online version of the original article can be found at http://dx.doi.org/ 10.1007/s12011-014-0213-3.

Yuan-Zhong Wang boletus@126.com

1 Institute of Medicinal Plants, Yunnan Academy of Agricultural Sciences, Kunming 650200, China

2 College of Resources and Environment, Yuxi Normal University, Yuxi 653100, China

3 College of Agronomy and Biotechnology, Yunnan Agricultural University, Kunming 650201, China 
Table 2 Arsenic in Laccaria mushrooms in literature

\begin{tabular}{|c|c|c|c|c|c|}
\hline Species & Site & $\begin{array}{l}\text { Number of } \\
\text { samples }^{\text {a }}\end{array}$ & Part & $\begin{array}{l}\text { Arsenic } \\
\text { concentration } \\
(\mathrm{mg} \mathrm{kg}-1 \mathrm{dw})\end{array}$ & Reference \\
\hline L. amethystina & Ljubljana, Slovenia & $3(>60)$ & Cap+stipe & $34-182$ & {$[18]$} \\
\hline L. amethystina & Ljubljana, Slovenia & $11(>220)$ & Cap & $41-182$ & {$[18]$} \\
\hline L. amethystina & Domzale, Slovenia & - & Cap & $109-200$ & {$[24]$} \\
\hline L. amethystina & Slivna and Voltji potok, Slovenia & $2(-)$ & Cap + stipe & 34,41 & {$[25]$} \\
\hline L. amethystina & North of Copenhagen, Denmark & $2(>20)$ & Cap + stipe & 23,77 & {$[20]$} \\
\hline L. amethystina & Hillerød, Denmark (contaminated) & $1(>10)$ & Cap + stipe & 1420 & {$[20]$} \\
\hline L. amethystina ( $\equiv$ L. laccata var. amethystina) & Nazareth, Belgium & 1 & Cap + stipe & 172 & {$[26]$} \\
\hline L. fraterna & Unknown & $4(-)$ & Cap + stipe & $11-30$ & {$[27]$} \\
\hline L. laccata & East Black Sea region, Turkey & 1 & Cap + stipe & 145 & {$[28]$} \\
\hline L. laccata (三L. affinis) & Reggio Emilia, Italy & $1(-)$ & Cap + stipe & 4.12 & {$[29]$} \\
\hline L. laccata & East Black Sea region, Turkey & $1(-)$ & Cap + stipe & 1.76 & {$[30]$} \\
\hline L. laccata $(\equiv$ L. amethystea $)$ & Slovenia & $8(-)$ & Cap & $25-129$ & {$[31]$} \\
\hline L. laccata & Slovenia & $9(-)$ & Cap & $11.5-33.4$ & {$[31]$} \\
\hline L. laccata $(\equiv$ L. laccata var. pallidifolia $)$ & Unknown & $2(-)$ & Cap + stipe & $0.66-4.3$ & {$[27]$} \\
\hline L. laccata $(\equiv$ L. amethystea $)$ & Hungary & $4(-)$ & Cap + stipe & $4-147$ & {$[19]$} \\
\hline L. laccata & Hungary & $4(-)$ & Cap + stipe & $1.8-16.4$ & {$[19]$} \\
\hline L. proxima & Slovenia & $6(-)$ & Cap & $0.8-4.3$ & [31] \\
\hline
\end{tabular}

The "dash" (-) symbol means without data

${ }^{a}$ Number of pooled samples (with number of all individuals) 